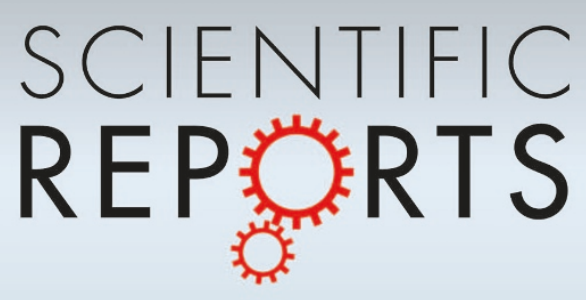

OPEN

SUBJECT AREAS:

FERROMAGNETISM

ELECTRONIC PROPERTIES AND

MATERIALS

MAGNETIC PROPERTIES AND

MATERIALS

Received

26 March 2014

Accepted

22 May 2014

Published

10 June 2014

Correspondence and requests for materials should be addressed to

Q.W. lqianwang2@ pku.edu.cn)

\section{Robust ferromagnetism in monolayer chromium nitride}

\author{
Shunhong Zhang' ${ }^{1}$ Yawei Li ${ }^{2}$, Tianshan Zhao' \& Qian Wang ${ }^{1,2}$ \\ ${ }^{1}$ Center for Applied Physics and Technology, College of Engineering, Peking University, Beijing 100871, China, ${ }^{2}$ Department of \\ Materials Science and Engineering, College of Engineering, Peking University, Beijing 100871, China.
}

Design and synthesis of two-dimensional (2D) materials with robust ferromagnetism and biocompatibility is highly desirable due to their potential applications in spintronics and biodevices. However, the hotly pursued 2D sheets including pristine graphene, monolayer $\mathrm{BN}$, and layered transition metal dichalcogenides are nonmagnetic or weakly magnetic. Using biomimetic particle swarm optimization (PSO) technique combined with $a b$ initio calculations we predict the existence of a $2 \mathrm{D}$ structure, a monolayer of rocksalt-structured $\mathrm{CrN}$ (100) surface, which is both ferromagnetic and biocompatible. Its dynamic, thermal and magnetic stabilities are confirmed by carrying out a variety of state-of-the-art theoretical calculations. Analyses of its band structure and density of states reveal that this material is half-metallic, and the origin of the ferromagnetism is due to $p$ - $d$ exchange interaction between the $\mathrm{Cr}$ and $\mathrm{N}$ atoms. We demonstrate that the displayed ferromagnetism is robust against thermal and mechanical perturbations. The corresponding Curie temperature is about $675 \mathrm{~K}$ which is higher than that of most previously studied 2D monolayers.

T wo dimensional (2D) materials have received intensive attention ${ }^{1-3}$ owing to the birth of graphene $e^{4}$. Materials in low dimensions can have electronic and magnetic properties that are fundamentally different from those of bulk phases. For instance, cubic boron nitride (BN) is an insulator whereas $\mathrm{BN}$ nanofilms can be intrinsically metallic ${ }^{5}$. Bulk $\mathrm{MoS}_{2}$ is an indirect band gap semiconductor while monolayer $\mathrm{MoS}_{2}$ has a direct bandgap of $1.90 \mathrm{eV}$, making it a promising material for nanoelectronics and valleytronics ${ }^{2,3}$. In this work, we explore the possibility of achieving highly spin-polarized ferromagnetism in chromium nitride (CrN) by altering its dimensionality.

Transition metal nitrides (TMNs) constitute a large family of materials with rich fundamental properties and vast technological applications. They have traditionally been used at extreme conditions of temperature and pressure due to their superhardness, high melting points, and outstanding corrosion resistance. In this family, $\mathrm{CrN}$, a prototypical TMN, is particularly interesting not only because it combines all the favorable properties of TMNs but also due to its high thermal stability, good electrical conductivity ${ }^{6}$, and especially its biocompatibility giving rise to its applications on medical implants and devices, such as artificial joints and cutting tools ${ }^{7}$. $\mathrm{CrN}$ has challenged both theoretical and experimental investigations ${ }^{8-17}$ for its rare "itinerant antiferromagnetism"11. Currently, two typical phases have been identified: the rocksalt-structured phase with $F m-3 m$ symmetry and paramagnetic (PM) order, and the orthogonal phase with Pnma symmetry and antiferromagnetic (AFM) order $^{8,11}$. Driven by magnetic stress induced lattice distortion, the former phase can transform into the latter at a very low external pressure ${ }^{10,13}$. However, neither the rocksalt-structured nor the orthogonal phase exhibits net magnetization despite remarkable local magnetic moments observed.

Previous studies have demonstrated that magnetism of nanomaterials differs from that of bulk materials in a fundamental way because both magnetic coupling and magnetic moments are strongly dependent on coordination number and dimensionality-related quantum confinements. For example, in nanoscale, the AFM coupling between $\mathrm{Cr}$ atoms can be switched to ferromagnetic (FM) by means of introducing nitrogen ${ }^{18}$ or oxygen atoms ${ }^{19}$. The magnetic coupling between the $\mathrm{Mn} / \mathrm{Cu}$ dopants in dilute magnetic semiconductors (DMSs) is highly dependent on dimensionality, namely, exhibiting FM coupling configuration in 1D GaN nanowires ${ }^{20-22}$ while having AFM coupling in the $2 \mathrm{D}$ thin films ${ }^{23}$, which implies the possibility of achieving ferromagnetism in $\mathrm{CrN}$ system by altering its dimensionality. What's more, even in the AFM phase of bulk $\mathrm{CrN}$ there exists ferromagnetic layers ${ }^{8,11}$. These inspired us to carry out the following study to seek a FM CrN system as it may have applications in spintronics as well as biotechnology. 


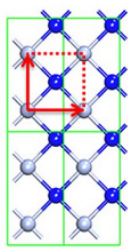

$Z=2$

(a)

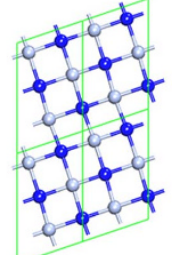

$\mathrm{Z}=3$

(b)

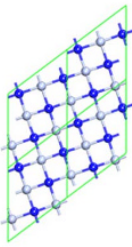

(c)

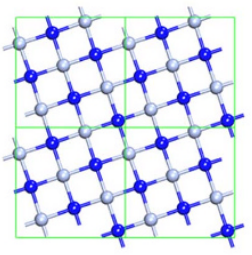

$Z=5$

(d)
Figure $1 \mid \mathrm{CrN}$ in 2D form. The 2D structures of $\mathrm{CrN}$ predicted by CALYPSO with respect to the number of formula units $\mathrm{Z}$ per supercell. Silver and blue balls represent $\mathrm{Cr}$ and $\mathrm{N}$ ions, respectively. For clarity all structures are shown in $2 \times 2$ supercells (green lines). The red lines denote the lattice basis vectors of the unit cell.

\section{Results}

Structure search. We first revisit $\mathrm{CrN}$ in bulk phase to test the methods that we used. Full relaxations of the lattices and atomic positions are performed for the rocksalt phase of $\mathrm{CrN}$ with both the FM and AFM coupling configurations of the $\mathrm{Cr}$ atoms, respectively. The FM configuration is found to be energetically less favorable than the AFM one by an energy difference of $210 \mathrm{meV}$, which is in good agreement with previous results ${ }^{6}$ as well as the fact that bulk $\mathrm{CrN}$ is an AFM material at low temperature. We then begin the study of $\mathrm{CrN}$ in two-dimensional form. A thorough exploration on the potential energy surface (PES) of $2 \mathrm{D} \mathrm{CrN}$ is needed to identify the thermodynamically stable structure. We perform unbiased structural searches by means of the CALYPSO (Crystal structure AnaLYsisby Particle Swarm Optimization) ${ }^{24}$ technique based on the biomimetic Particle Swarm Optimization (PSO) algorithm, which has successfully predicted a series of $2 \mathrm{D}$ crystals $^{25-31}$ with a considerably high efficiency. Without any priori structural information, our blind structure search converges to a planar oneatomic-thick structure with an optimized lattice constant of $2.82 \AA$ and $\mathrm{Cr}-\mathrm{N}$ bond length of $1.99 \AA$, irrespective to the choice of supercell size $Z$ ( $Z$ represent the number of formula units in a supercell,) as shown in Figure 1 (See the Supplementary Information for details on the structure search).

Preferred Magnetic Configuration. The predicted 2D CrN structure turns out to be surprisingly simple, which can be viewed as a monolayer of the rocksalt-structured $\mathrm{CrN}(100)$ surface. To search for the magnetic ground state of this monoatomic thick structure, we calculate the spin polarization energy ${ }^{32}$, namely, the energy difference between the nonmagnetic and magnetic configurations. Our calculations yield a large energy gain of $1.6 \mathrm{eV} /$ unit cell upon spin polarization, indicating that the magnetic configuration is highly preferred and the monolayer $\mathrm{CrN}$ might be a good magnetic material. In the spin polarized configuration, the bond length of $\mathrm{Cr}-\mathrm{N}$ is $1.99 \AA$, which is $4.7 \%$ larger than that of the nonmagnetic configuration. Each unit cell carries a net magnetic moment of $3.00 \mu_{\mathrm{B}}$, which arises mainly from the Cr $3 d$ orbitals $\left(3.19 \mu_{\mathrm{B}}\right)$ and the neighboring $\mathrm{N}$ atom is polarized antiferromagnetically with a magnetic moment of -0.37 $\mu_{\mathrm{B}}$. This is because the $\mathrm{Cr}$ ions in this nanosheet have valance state of + III based on the chemical stoichiometry and the electronic configuration of $\mathrm{Cr}^{3+}$ is $3 d^{3}$. We then consider a low spin configuration by constraining the total magnetic moment of a unit cell to be $1 \mu_{\mathrm{B}}$. The calculated total energy of the low spin configuration is $0.95 \mathrm{eV}$ higher than that of the high-spin state. In addition, we find the lattice constants undergo a expansion from $2.70 \AA \times 2.70 \AA$ (the NM state) to $2.73 \AA \times 2.75 \AA$ (low-spin state) to $2.82 \AA \times 2.82 \AA$ (high-spin state), and the $\mathrm{Cr}$ - $\mathrm{N}$ bond length changes from $1.90 \AA$ to $1.94 \AA$ to $1.99 \AA$, accordingly. This indicates that spin polarization can effectively release the strain and stabilize the system.

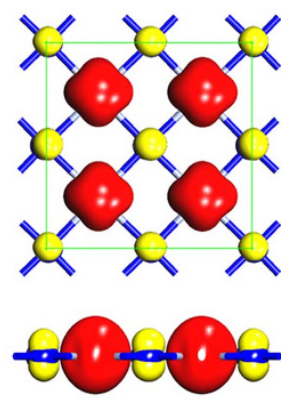

FM: 0 meV

(a)
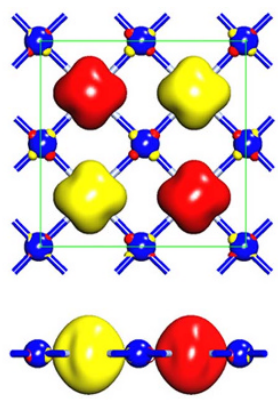

AFM: $632 \mathrm{meV}$

(b)
Figure 2| Magnetic coupling configurations of the monolayer CrN. Top and side views of the optimized geometry and spin charge density isosurface (isovalue: $0.2 \mathrm{e} / \AA^{3}$ ) of (a) the FM and (b) AFM configurations of the monolayer $\mathrm{CrN}$. Red and yellow isosurfaces correspond to spin up and spin down charge density, respectively. Green squares denote the $2 \times 2$ supercell.

To further determine the most preferable magnetic coupling between the $\mathrm{Cr}$ atoms in this predicted $\mathrm{CrN}$ nanosheet, we perform spin polarized total energy calculations for the two typical collinear magnetic configurations, namely, the FM and AFM states, within a 2 $\times 2$ supercell. The FM state is found to be $632 \mathrm{meV}$ lower in energy than the AFM one, indicating that the monolayer CrN is FM. Such a magnitude of energy difference is observable in experiments. Figure 2 shows the spin charge density distributions $\left(\rho_{\uparrow}-\rho_{\downarrow}\right)$ for both the FM and AFM coupling configurations. We note that large spin charge accumulates around the $\mathrm{Cr}^{3+}$ sites in both the two cases, resulting in large local magnetic moments. However, the spin charge distribution of the N $2 p$ electrons appears differently: In the FM ground state, the $\mathrm{N} 2 p$ electrons are strongly spin polarized antiferromagnetically with the $\mathrm{Cr}$ ions, while in the AFM configuration the net spin polarization in the ligands is negligible, resembling that in its bulk counterpart ${ }^{16}$.

Dynamic Stability. To ensure that the CrN nanosheet can be practically utilized as a FM material, we first examine its dynamical stability by calculating its phonon spectra. The Phonon dispersion and frequency density of states (PhDOS) are displayed in Figure 3(a). All vibrational modes are found to be real in the whole Brillouin Zone, indicating this structure is dynamically stable and can exist as a freestanding $2 \mathrm{D}$ crystal.

Thermal Stability. We then examine the thermal stability of the monolayer $\mathrm{CrN}$. We construct a large supercell of $6 \times 6$ containing 36 formula units to minimize the constraint induced by periodicity. Ab initio molecular dynamics (MD) simulations $s^{33-35}$ are performed at $300 \mathrm{~K}$ with a time step of $1 f s$ using Nosé heat bath scheme $^{36}$ to investigate whether the FM monolayer is thermally stable at room temperature. The atomic configuration of the monolayer remains nearly intact after heated for more than 6 picoseconds. The variations of the total potential energy and total magnetic moment with respect to simulation time are plotted in Figure 3(b), showing that the monolayer is thermally stable as both of them remain almost invariant during the simulation. We further gradually increase heating temperature and find the structure can survive up to $600 \mathrm{~K}$. MD simulations are also carried out for a $4 \sqrt{2} \times$ $4 \sqrt{ } 2$ supercell containing 32 formula units. We find, again, neither structural reconstruction nor magnetic phase transition occurs when the structure is heated at room temperature (see Figure S2 in Supplementary Information for details). These results demonstrate that the FM monolayer CrN, once synthesized, is stable and can stay robust at room temperature. 


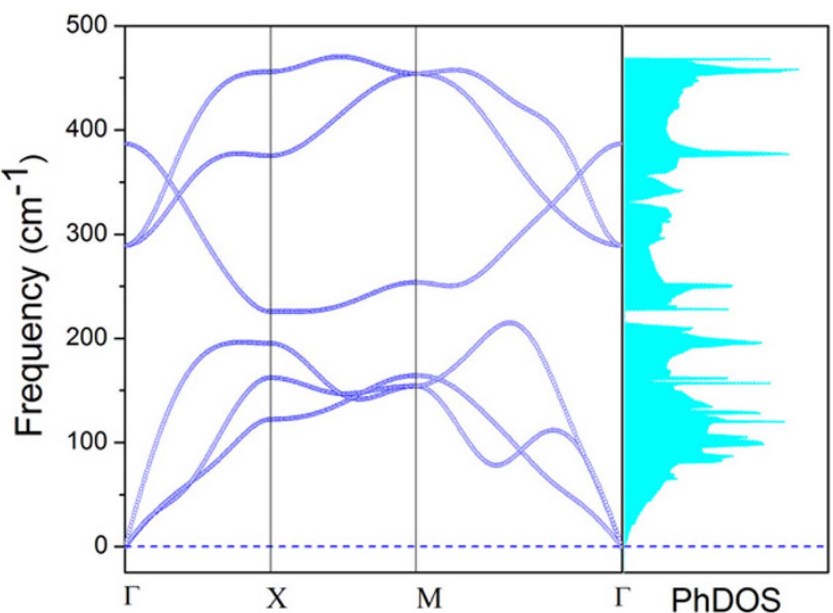

(a)

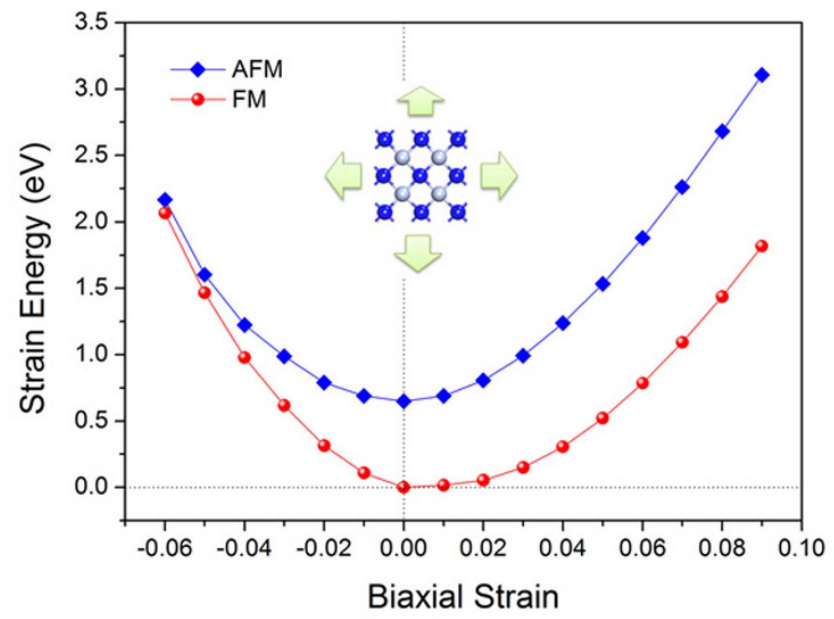

(c)

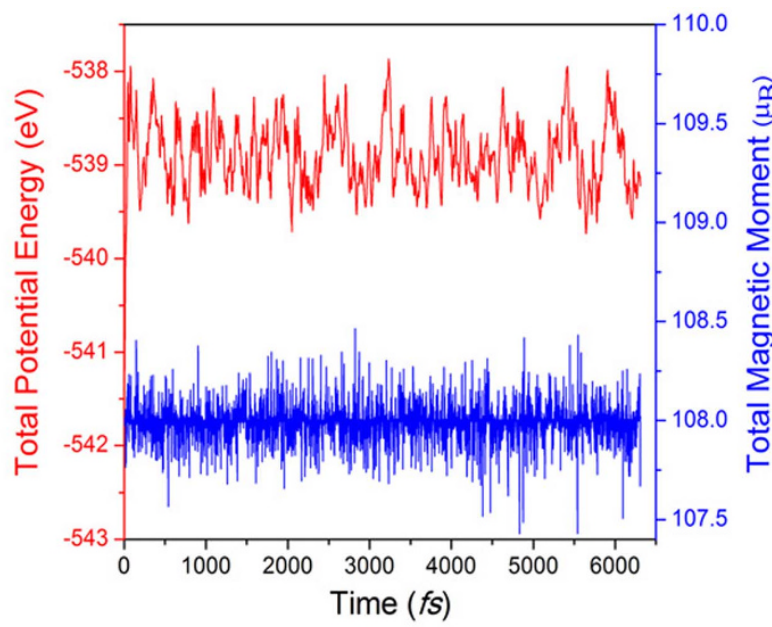

(b)
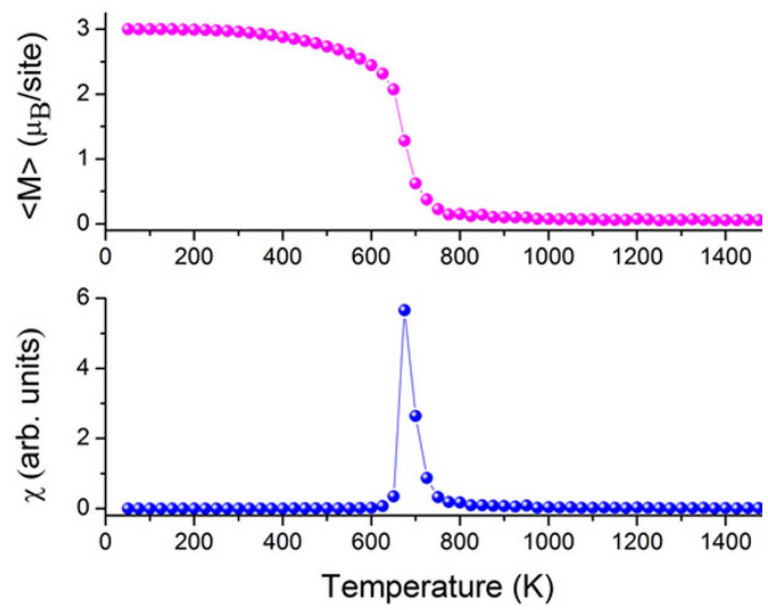

(d)

Figure $3 \mid$ Structural and magnetic stability of the monolayer CrN. (a) Phonon dispersion and frequency DOS (in arbitrary unit) of the monolayer CrN.The high symmetry q point path in the Brillouin Zone is $\Gamma(0,0) \rightarrow \mathrm{X}(1 / 2,0) \rightarrow \mathrm{M}(1 / 2,1 / 2) \rightarrow \Gamma(0,0)$. (b) Variations of the total potential energy and total magnetic moment with respect to simulation time. (c)Strain energy of the FM and AFM configurations under the biaxial strains. The inset shows the $2 \times 2$ supercell used to calculate the strain energy. (d) Variations of the average magnetic moment per unit cell and magnetic susceptibility $(\chi)$ with respect to temperature.

To check if any possible structure reconstruction or buckling pattern $^{37}$ could happen, we create a single-layer PbO-type structured $\mathrm{CrN}$ which is ubiquitous in many layered crystals ${ }^{38}$. We find, upon full relaxation, the PbO-type structure of $\mathrm{CrN}$ spontaneously converges to the planar monolayer sheet, indicating the outstanding stability of this CrN structure.

Magnetic Stability. We further study the stability of the FM order of the monolayer $\mathrm{CrN}$ against the in-plane strains. $\mathrm{CrN}$ thin films, in reality, usually grow on certain substrates like $\mathrm{MgO}(100)$ surface $^{9,39}$. The synthesized thin films might endure some external strain, either compressive or tensile stain, due to the possible lattice mismatch. We calculate the strain energy for both the FM and AFM configurations under the isotropic biaxial in-plane strain. The results are plotted in Figure 3(c). Although the exchange energy decreases with the compressive biaxial strain and increases with the tensile strain, the FM state is found to always lie lower in energy than the AFM configuration in a wide range of the biaxial strains from $-6 \%$ to $8 \%$. This indicates that the FM order in the monolayer $\mathrm{CrN}$ can remain virtually undisturbed from the in-plane strains. A noteworthy trend in the strain energy curves is that the FM state becomes less competitive upon increasing the compressive biaxial strain, which is reminiscent of previous prediction on the bond length contraction induced AFM ordering in the Mn-doped GaN thin films $\mathrm{s}^{23}$.

Estimation of Curie temperature. Having confirmed the structural and magnetic stability, we then quantitatively study the stability of the FM ordering against thermal perturbation by estimating its Curie temperature. To this end we carry out Metropolis Monte Carlo (MC) simulations based on 2D Ising mode ${ }^{40}$. According to the classical Heisenberg model, the Hamiltonian of a 2D lattice can be expressed as $\hat{H}=-\sum_{i, j} J \hat{m}_{i} \hat{m}_{j}$, where $m_{i}$ corresponds to the magnetic moments (in $\mu_{\mathrm{B}}$ ) at site $i$ (each unit cell is treated as one site, and for each site four spin states are considered, namely, two high spin sates, $S=3 / 2$ and $S=-3 / 2$, and two low spin states, $S=1 / 2$ and $S=-1 / 2$, and $J$ refers to the Heisenberg direct exchange parameter, which can be obtained by $J=(1 / 8) E_{e x} / 2 m^{2}$, where $E_{\text {ex }}$ represents the exchange energy of the AFM and FM states in the $2 \times 2$ supercell. The factors of $1 / 8$ and $1 / 2$ correspond to the eight exchange pairs in the supercell and the double count of each pair in the summation, 


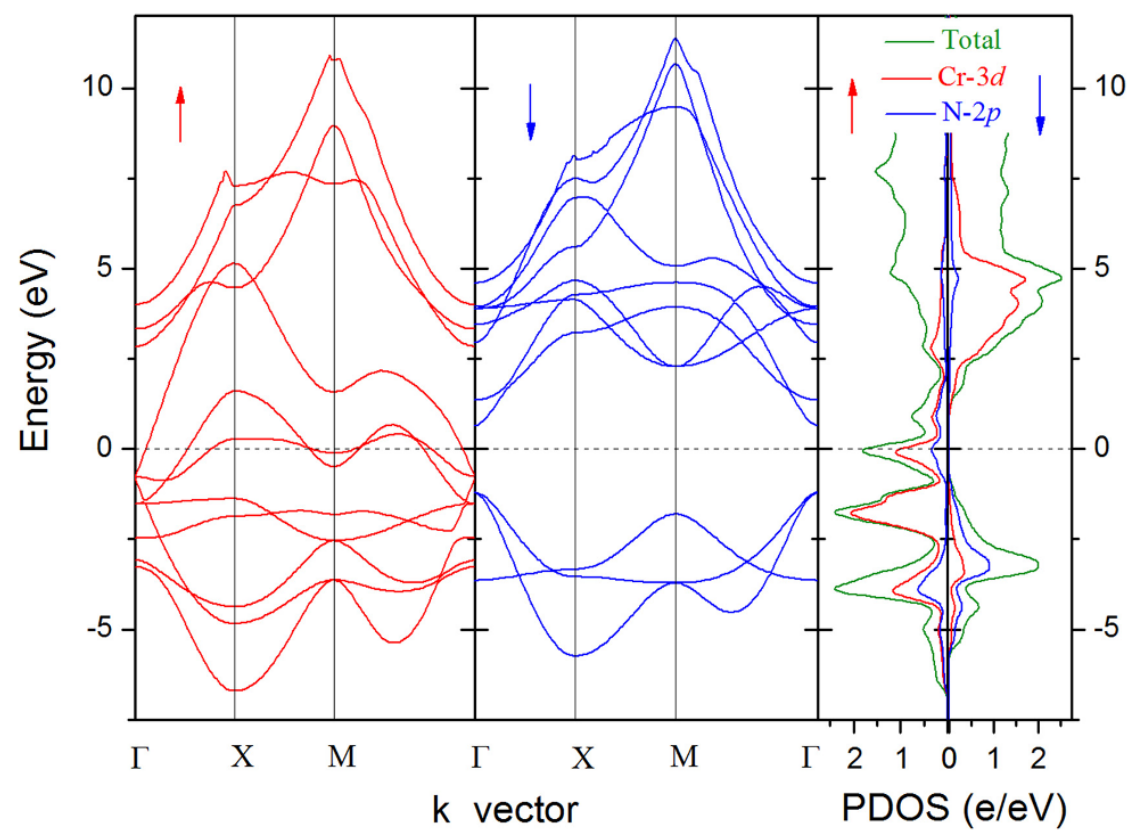

Figure 4 Electronic properties. Spin-polarized electronic band structure (Red: spin up channel; Blue: spin down channel) and PDOS of the monolayer $\mathrm{CrN}$. The Fermi level is shifted to $0 \mathrm{eV}$.

respectively. Substituting $E_{\mathrm{ex}}=632 \mathrm{meV}$ and $m=3$ into the formula, we get $J=4.39 \mathrm{meV}$. For simplicity, only the nearest neighbor exchange interaction is taken into account in the Hamiltonian. In order to reduce the periodic constraints, a $50 \times$ 50 supercell is used to mimic the monolayer sheet. The variations of average magnetic moment per unit cell and magnetic susceptibility with respect to temperature are plotted in Figure 3(d). We note that the magnetic moment remains in the high spin state in the lowtemperature range and then drastically drops to near zero and the magnetic susceptibility appears a sharp peak at a critical temperature, showing a thermal-induced FM-to-PM phase transition. The Curie temperature is estimated to be around $675 \mathrm{~K}$, which is higher than that of most previously studied $2 \mathrm{D}$ systems ${ }^{34,35,40-48}$. This implies that ferromagnetism in the monolayer $\mathrm{CrN}$ is robust and can be detected at room temperature.

Electronic Properties. Bulk $\mathrm{CrN}$ is known as a "borderline Mott insulator"14, to investigate the electronic properties of the monolayer sheet, we calculate the spin-polarized electronic band structure. The results are plotted in Figure 4 . We note although the band dispersion relationships in both spin channels are similar, it exhibits metallic feature in the spin-up channel characterized by the Fermi level crossing the partially occupied valance bands in the Brillouin zone and semiconducting feature in spin-down channel, indicating that the monolayer $\mathrm{CrN}$ is half-metallic. We further calculate the total density of states (DOS) and orbital resolved partial DOS at the Cr and $\mathrm{N}$ sites to explore the origin of the halfmetallicity and ferromagnetism. There is a remarkable DOS peak at the Fermi level which is only contributed by the spin up channel. The partial DOS shows an explicit spin splitting in both the Cr $3 d$ and $\mathrm{N} 2 p$ states. The overlap between the Cr $3 d$ and N $2 p$ orbitals leads to the significant DOS near the Fermi level in spin-up channel, thus results in the half-metallicity as well as the ferromagnetism. Observably, the $\mathrm{N} 2 p$ orbitals play key role in mediating the magnetic coupling between the $\mathrm{Cr}$ atoms.

Bilayer Stacking: structural and magnetic configurations. So far, we have established the viability of realizing robust ferromagnetism in the monolayer $\mathrm{CrN}$. It would be intriguing to see how the structural and magnetic configurations change when the two CrN monolayers are stacked to form bilayer $\mathrm{CrN}$. To address this concern we build two typical bilayer models, namely, AA and AB stacking, as shown in Figure 5. Our calculated results show that the AB stacking is energetically more favorable than the AA stacking. The binding energy between the two monolayers is $1.428 \mathrm{eV}$ and $0.641 \mathrm{eV}$, and the optimized interlayer distance is $2.09 \AA$ and $3.18 \AA$ for the $\mathrm{AB}$ and AA stacking patterns, respectively, showing different bonding characters, namely, weak interaction in the AA stacking and a strong bonding with a hybrid ionic and covalent feature in the $\mathrm{AB}$ stacking. This is different from the situations of graphene and $\mathrm{BN}$ sheets which have weak interactions between layers, but is similar to the case of Fe-phthalocyanine sheets, where the interaction between the two layers is strong. However, a single Fe-phthalocyanine sheet can be synthesized on an insulating substrate ${ }^{49}$, where the $\mathrm{Fe}$ phthalocyanine sheet only weakly interacts with the substrate ${ }^{44}$. Therefore, we can expect that our predicted $\mathrm{CrN}$ sheet could be grown on some insulating substrates. Since ferromagnetism of the monolayer $\mathrm{CrN}$ is energetically robust against antiferromagnetism, for simplicity we first assume that the intra-layer coupling is FM like a freestanding monolayer, and for each stacking pattern we consider two inter-layer couplings, namely, FM and AFM. We relax the lattice and atomic positions with inclusion of Grimme's potential ${ }^{50}$ to compensate for missing van der Waals interactions in standard

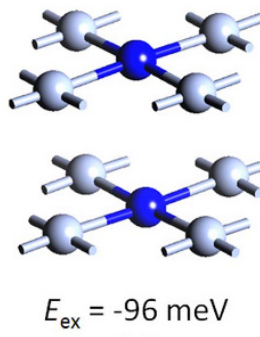

(a)

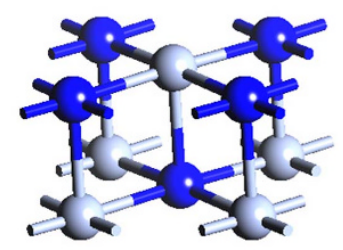

$E_{\mathrm{ex}}=209 \mathrm{meV}$

(b)
Figure $5 \mid$ Stacking pattern of Bilayer CrN. (a) AA stacking and (b) AB stacking. The exchange energies (define as energy difference between the AFM and FM configurations) of possible magnetic configurations are presented. Here FM and AFM refer to inter-layer magnetic coupling. 
DFT calculations. Total energy calculations show that the $A B$ stacking FM coupling configuration has the lowest energy among all the considered bilayer models. We then enlarge the $\mathrm{AB}$ stacking model to a $2 \times 2$ supercell to examine the intra-layer magnetic coupling. Three possible magnetic coupling configurations: FM (both intra- and inter layer couplings are FM), partial AFM (intralayer FM and inter-layer AFM), and AFM (both intra- and interlayer couplings are AFM) are considered. The calculated total energy relative to the FM state for the partial AFM and AFM state are $248 \mathrm{meV}$ and $209 \mathrm{meV}$, respectively. This confirms that the bilayer $\mathrm{CrN}$ is also FM. Therefore, we can conclude that the CrN monolayer, either in freestanding form or stacked in energetically favorable patterns, can exhibit robust ferromagnetism.

\section{Discussion}

To better understand the ferromagnetism of the monolayer $\mathrm{CrN}$, it is worth recalling the following facts. (1) Chromium, due to its unique electronic configuration of [Ar] $3 d^{5} 4 s^{1}$ and the small energy gap between the $3 d$ and $4 s$ orbitals, can exist in different oxidation states, namely, $0,+2,+3$, and +6 , which can be used to tune the structural and magnetic properties of $\mathrm{Cr}$-containing materials by changing its bonding feature. (2) In a $\mathrm{Cr}_{2}$ dimer, $\mathrm{Cr}$ atom binds strongly with the other $\mathrm{Cr}$ atom and the resulting sextuple bonding yields a large binding energy $(1.44 \mathrm{eV})$ and a very short bond length $(1.68 \AA)$. Small clusters of $\mathrm{Cr}$ containing five atoms or fewer are all $\mathrm{AFM}^{51}$. In the bulk phase, $\mathrm{Cr}$ is also $\mathrm{AFM}^{52}$. However, the structures and magnetic properties of these small clusters can be drastically modified by doping a nitrogen atom ${ }^{18}$, where the nearest-neighbor $\mathrm{Cr}$ atoms are coupled antiferromagnetically to the $\mathrm{N}$ atom and hence ferromagnetically with each other. Similarly, in the ground state of the monolayer $\mathrm{CrN}$, the two nearest $\mathrm{Cr}$ atoms are too far apart to allow significant direct overlap of the unpaired electron wave functions, the coupling involves the $\mathrm{N} 2 p$ orbitals polarized antiferromagnetically to the $3 d$ orbitals of $\mathrm{Cr}$ atoms. This lowers the energy of the system and makes the $\mathrm{Cr}$ atoms couple ferromagnetically to each other. Thus, the mechanism responsible for the FM coupling in the monolayer $\mathrm{CrN}$ can be classified as the $p$ - $d$ exchange mechanism ${ }^{53}$. (3) To achieve highly spin polarized ferromagnetism, usually it is necessary to introduce transition metal atoms. The observed magnetism originated from $2 p$ electrons in the currently studied systems like porous graphene or hydrogenated ${ }^{35}$ or fluorinated graphene is weak. Among $3 d$ transition metals, $\mathrm{Cr}$ and $\mathrm{Mn}$ have been widely used as dopants in semiconductors such as $\mathrm{GaN}$ or $\mathrm{GaAs}$ or $\mathrm{ZnO}$ to introduce magnetism due to their unique electronic configurations. However, it is challenging to regularly dock $\mathrm{Cr}$ or $\mathrm{Mn}$ atoms into the bulk phases and even on the 2D structures because of the clustering of TM atoms resulting from strong $d$ - $d$ orbital interactions. Recent experimental advances have demonstrated that $2 \mathrm{D}$ phthalocyaninebased organometallic (labeled as TM-Pc) sheets ${ }^{40,49}$ and tetragonal transition-metal-based 7,7,8,8-tetracyanoquinodimethane molecule coordination sheets (labeled as TM@TCNQ) ${ }^{54}$ have been successfully synthesized with the TM atoms regularly and separately distributed, providing the possibility of synthesizing and the $2 \mathrm{D} \mathrm{CrN}$ sheet experimentally and detecting the ferromagnetism. (4) Different from $\mathrm{Mn}$-containing 2D sheets where $\mathrm{Mn}^{2+}$ and $\mathrm{Mn}^{3+}$ are neurotoxic ${ }^{55}$, as they can enter the brain by crossing the blood-brain barrier and cause permanent neurological disorder, the explored 2D CrN sheet is biocompatible, where trivalent chromium $\left(\mathrm{Cr}^{3+}\right)$ in $\mathrm{CrN}$ is biologically active ${ }^{56}$, widely found in food and supplements, meanwhile nitrogen can also improve biocompatibility significantly ${ }^{57}$.

\section{Summary}

Motivated by design of novel 2D magnetic materials and inspired by the chemical control of magnetic coupling in molecules and clusters $^{18,19}$, a comprehensive theoretical study on geometry, stability, electronic properties and ferromagnetism of the monolayer $\mathrm{CrN}$ sheet is performed. We find CrN, a well-known AFM or PM material in bulk phases, can become spontaneously FM when sliced into oneatom-thick nanosheet. The switching between AFM or PM to FM couplings is brought by tailoring the dimensionality and the origin of ferromagnetism is ascribed to $p$ - $d$ exchange mechanism. The welldefined atomic configuration and intrinsic ferromagnetism of the monolayer $\mathrm{CrN}$ suggest it is superior to other $2 \mathrm{D}$ magnetic materials: (1) Unlike other 2D Cr-containing sheets such as Cr-Pc and TM@ TCNQ monolayers, which are AFM, the CrN nanosheet predicted here is both stable and FM, and the ferromagnetism can be sustained against mechanical and thermal perturbations; (2) Unlike other TMbased 2D materials which needs external stimulus like chemical functionalization $^{34,35,58}$, carrier injection ${ }^{34,44}$ or external $\operatorname{strain}^{42}$ to switch on or enhance the FM order, the $2 \mathrm{D} \mathrm{CrN}$ sheet possesses intrinsic ferromagnetism with a very high Curie temperature of $675 \mathrm{~K}$, which is much higher than that of previously reported $150 \mathrm{~K}$ and $140 \mathrm{~K}$ for $\mathrm{Mn}-\mathrm{Pc}$ sheet ${ }^{40}$ and $\mathrm{MnO}_{2}$ monolayer ${ }^{42}$, respectively; (3) Unlike conventional $\mathrm{Cr}$ or Mn doped DMSs where semiconductors are used as matrix to host the magnetic dopants, and magnetism, in most cases, comes from the clustering of TM atoms, in the monolayer $\mathrm{CrN}, \mathrm{Cr}$ ions are individually held at the specific sites in a $2 \mathrm{D}$ crystal structure, and couple instrically ferromagnetically with each other. The Curie temperature is much higher than the highest $T_{c}$ value achieved experimentally in Mn-doped GaAs. In addition, $\mathrm{CrN}$ thin film has been widely used as a coating material owing to its outstanding corrosion-resistance ${ }^{10}$. Therefore, the displayed high stability, robust ferromagnetism and the intriguing biocompatibility make this monolayer $\mathrm{CrN}$ sheet a very promising candidate for applications in spintronics, biodevices and functional coating techniques. We hope that the present study will stimulate further experimental effort in this direction.

\section{Methods}

Our first principles calculations are based on spin-polarized Density Functional Theory (DFT). All-electron Projector Augmented Wave (PAW) method implemented in Vienna $A b$ initio Simulation Package (VASP) ${ }^{59}$ is used to investigate the ground state structural, electronic and magnetic properties of $\mathrm{CrN}$ in the bulk and monolayer phases. For $\mathrm{N}$ and $\mathrm{Cr}, 2 s^{2} 2 p^{5}$ and $3 d^{5} 4 s^{1}$ electrons are treated as valence electrons, respectively. Considering the localization character of $\mathrm{Cr} 3 d$ electrons, $\mathrm{GGA}+U$ formalism is adopted to describe the strong on-site Coulomb interaction. We choose the simplified (rotationally invariant) approach due to Dudarev et al. ${ }^{60}$, which involves only one parameter, namely, the effective $U(U-J$, where $U$ is the onsite Coulomb repulsion and $J$ is the onsite Hund exchange coupling). We set the effective $U$ value to be $3 \mathrm{eV}$, which has been widely used in both bulk ${ }^{14,17,61,62}$ and lowdimensional ${ }^{40,44,63,64}$ systems that contain Cr or other $3 d$ transition metal atoms and provided reasonable results. $2 \mathrm{D}$ structure searches are carried out using the CALYPSO methodology ${ }^{24-26}$ in combination with VASP for local optimizations and total energy calculations. Phonon properties are calculated using Density Functional Perturbation Theory (DFPT) ${ }^{65}$ implemented in the Quantum ESPRESSO suite ${ }^{66} . A b$ Initio Molecular Dynamics (AIMD) simulations are performed to examine the thermal stability. Detailed computational procedures are provided in the Supplementary Information.

1. Butler, S. Z. et al. Progress, Challenges, and Opportunities in Two-Dimensional Materials Beyond Graphene. ACS Nano 7, 2898-2926 (2013).

2. Xu, M., Liang, T., Shi, M. \& Chen, H. Graphene-Like Two-Dimensional Materials. Chem. Rev. 113, 3766-3798 (2013).

3. Chhowalla, M. et al. The chemistry of two-dimensional layered transition metal dichalcogenide nanosheets. Nat. Chem. 5, 263-275 (2013).

4. Novoselov, K. S. et al. Electric Field Effect in Atomically Thin Carbon Films. Science 306, 666-669 (2004).

5. Zhang, Z. \& Guo, W. Intrinsic Metallic and Semiconducting Cubic Boron Nitride Nanofilms. Nano Lett. 12, 3650-3655 (2012).

6. Weimer, A. W. Carbide, nitride and boride materials synthesis and processing (Springer, 1997).

7. Fuentes, G. G. et al. Recent advances in the chromium nitride PVD process for forming and machining surface protection. J. Mater. Process. Technol. 167, 415-421 (2005).

8. Corliss, L. M., Elliott, N. \& Hastings, J. M. Antiferromagnetic Structure of CrN. Phys. Rev. 117, 929-935 (1960).

9. Inumaru, K., Koyama, K., Imo-oka, N. \& Yamanaka, S. Controlling the structural transition at the Néel point of CrN epitaxial thin films using epitaxial growth. Phys. Rev. B 75, 054416 (2007). 
10. Rivadulla, F. et al. Reduction of the bulk modulus at high pressure in CrN. Nat. Mater. 8, 947-951 (2009).

11. Bhobe, P. A. et al. Evidence for a Correlated Insulator to Antiferromagnetic Metal Transition in CrN. Phys. Rev. Lett. 104, 236404 (2010).

12. Filippetti, A., Pickett, W. E. \& Klein, B. M. Competition between magnetic and structural transitions in CrN. Phys. Rev. B 59, 7043-7050 (1999).

13. Filippetti, A. \& Hill, N. A. Magnetic Stress as a Driving Force of Structural Distortions: The Case of CrN. Phys. Rev. Lett. 85, 5166-5169 (2000).

14. Herwadkar, A. \& Lambrecht, W. R. L. Electronic structure of CrN: A borderline Mott insulator. Phys. Rev. B 79, 035125 (2009).

15. Alling, B., Marten, T. \& Abrikosov, I. A. Questionable collapse of the bulk modulus in CrN. Nat. Mater. 9, 283-284 (2010).

16. Botana, A. S., Tran, F., Pardo, V., Baldomir, D. \& Blaha, P. Electronic structure of CrN: A comparison between different exchange correlation potentials. Phys. Rev. B 85, 235118 (2012).

17. Steneteg, P., Alling, B. \& Abrikosov, I. A. Equation of state of paramagnetic CrN from ab initio molecular dynamics. Phys. Rev. B 85, 144404 (2012).

18. Wang, Q., Sun, Q., Rao, B. K., Jena, P. \& Kawazoe, Y. Nitrogen-induced magnetic transition in small chromium clusters. J. Chem. Phys. 119, 7124-7130 (2003).

19. Tono, K., Terasaki, A., Ohta, T. \& Kondow, T. Chemical Control of Magnetism: Oxidation-Induced Ferromagnetic Spin Coupling in the Chromium Dimer Evidenced by Photoelectron Spectroscopy. Phys. Rev. Lett. 90, 133402 (2003).

20. Wang, Q., Sun, Q. \& Jena, P. Ferromagnetism in Mn-Doped GaN Nanowires. Phys. Rev. Lett. 95, 167202 (2005).

21. Xiang, H. J. \& Wei, S.-H. Enhanced Ferromagnetic Stability in Cu Doped Passivated GaN Nanowires. Nano Lett. 8, 1825-1829 (2008).

22. Wang, Q., Sun, Q., Jena, P. \& Kawazoe, Y. Ferromagnetic GaN-Cr Nanowires. Nano Lett. 5, 1587-1590 (2005).

23. Wang, Q., Sun, Q., Jena, P. \& Kawazoe, Y. Antiferromagnetic Coupling Driven by Bond Length Contraction near the $\mathrm{Ga}_{1-\mathrm{x}} \mathrm{Mn}_{\mathrm{x}} \mathrm{N}$ Film Surface. Phys. Rev. Lett. 93 , 155501 (2004).

24. Wang, Y., Lv, J., Zhu, L. \& Ma, Y. Crystal structure prediction via particle-swarm optimization. Phys. Rev. B 82, 094116 (2010).

25. Luo, X. et al. Predicting Two-Dimensional Boron-Carbon Compounds by the Global Optimization Method. J. Am. Chem. Soc. 133, 16285-16290 (2011).

26. Wang, Y. et al. An effective structure prediction method for layered materials based on 2D particle swarm optimization algorithm. J. Chem. Phys. 137, 224108 (2012).

27. Wu, X. et al. Two-Dimensional Boron Monolayer Sheets. ACS nano 6, 7443-7453 (2012).

28. Luo, X. et al. Two-Dimensional Superlattice: Modulation of Band Gaps in Graphene-Based Monolayer Carbon Superlattices. J. Phys. Chem. Lett. 3, 3373-3378 (2012)

29. Yu, X., Li, L., Xu, X.-W. \& Tang, C.-C. Prediction of Two-Dimensional Boron Sheets by Particle Swarm Optimization Algorithm. J. Phys. Chem. C. 116, 20075-20079 (2012).

30. Zhou, L.-J., Zhang, Y.-F. \& Wu, L.-M. SiC 2 Siligraphene and Nanotubes: Novel Donor Materials in Excitonic Solar Cells. Nano Lett. 13, 5431-5436 (2013).

31. Dai, J., Zhao, Y., Wu, X., Yang, J. \& Zeng, X. C. Exploration of Structures of TwoDimensional Boron-Silicon Compounds with $s p^{2}$ Silicon. J. Phys. Chem. Lett. 4, 561-567 (2013).

32. Peng, H. et al. Origin and Enhancement of Hole-Induced Ferromagnetism in First-Row $d^{0}$ Semiconductors. Phys. Rev. Lett. 102, 017201 (2009).

33. Du, A., Sanvito, S. \& Smith, S. C. First-Principles Prediction of Metal-Free Magnetism and Intrinsic Half-Metallicity in Graphitic Carbon Nitride. Phys. Rev. Lett. 108, 197207 (2012).

34. Kan, E. et al. Two-Dimensional Hexagonal Transition-Metal Oxide for Spintronics. J. Phys. Chem. Lett. 4, 1120-1125 (2013).

35. Zhou, J. et al. Ferromagnetism in Semihydrogenated Graphene Sheet. Nano Lett. 9, 3867-3870 (2009).

36. Nosé, S. A unified formulation of the constant temperature molecular dynamics methods. J. Chem. Phys. 81, 511-519 (1984).

37. Zhang, Z., Liu, X., Yakobson, B. I. \& Guo, W. Two-Dimensional Tetragonal TiC Monolayer Sheet and Nanoribbons. J. Am. Chem. Soc. 134, 19326-19329 (2012).

38. Hsu, F.-C. et al. Superconductivity in the PbO-type structure $\alpha$-FeSe. Proc. Natl. Acad. Sci. 105, 14262-14264 (2008).

39. Constantin, C., Haider, M. B., Ingram, D. \& Smith, A. R. Metal/semiconductor phase transition in chromium nitride(001) grown by rf-plasma-assisted molecular-beam epitaxy. Appl. Phys. Lett. 85, 6371-6373 (2004).

40. Zhou, J. \& Sun, Q. Magnetism of Phthalocyanine-Based Organometallic Single Porous Sheet. J. Am. Chem. Soc. 133, 15113-15119 (2011).

41. Zhou, J., Wang, Q., Sun, Q. \& Jena, P. Intrinsic ferromagnetism in twodimensional carbon structures: Triangular graphene nanoflakes linked by carbon chains. Phys. Rev. B 84, 081402 (2011).

42. Kan, M., Zhou, J., Sun, Q., Kawazoe, Y. \& Jena, P. The Intrinsic Ferromagnetism in a $\mathrm{MnO}_{2}$ Monolayer. J. Phys. Chem. Lett. 4, 3382-3386 (2013).

43. Li, X., Zhou, J., Wang, Q., Kawazoe, Y. \& Jena, P. Patterning Graphitic C-N Sheets into a Kagome Lattice for Magnetic Materials. J. Phys. Chem. Lett. 4, 259-263 (2013).
44. Zhou, J. \& Sun, Q. Carrier induced magnetic coupling transitions in phthalocyanine based organometallic sheet. Nanoscale 6, 328-333 (2013)

45. Giesbers, A. J. M. et al. Interface-Induced Room-Temperature Ferromagnetism in Hydrogenated Epitaxial Graphene. Phys. Rev. Lett. 111, 166101 (2013).

46. Zhang, C.-W. \& Yan, S.-S. First-Principles Study of Ferromagnetism in TwoDimensional Silicene with Hydrogenation. J. Phys. Chem. C. 116, 4163-4166 (2012).

47. Kan, M., Adhikari, S. \& Sun, Q. Ferromagnetism in $\mathrm{MnX}_{2}(\mathrm{X}=\mathrm{S}$, Se) Monolayers. Phys. Chem. Chem. Phys. 16, 4990-4994 (2014).

48. Tan, J., Li, W., He, X. \& Zhao, M. Stable ferromagnetism and half-metallicity in two-dimensional polyporphyrin frameworks. RSC Adv. 3, 7016-7022 (2013).

49. Abel, M., Clair, S., Ourdjini, O., Mossoyan, M. \& Porte, L. Single Layer of Polymeric Fe-Phthalocyanine: An Organometallic Sheet on Metal and Thin Insulating Film. J. Am. Chem. Soc. 133, 1203-1205 (2010).

50. Grimme, S. Semiempirical GGA-type density functional constructed with a long range dispersion correction. J. Comput. Chem. 27, 1787-1799 (2006).

51. Cheng, H. \& Wang, L.-S. Dimer Growth, Structural Transition, and Antiferromagnetic Ordering of Small Chromium Clusters. Phys. Rev. Lett. 77, 51-54 (1996).

52. Fawcett, E. Spin-density-wave antiferromagnetism in chromium. Rev. Mod. Phys 60, 209-283 (1988).

53. Mizokawa, T. \& Fujimori, A. $p$ - $d$ exchange interaction for transition-metal impurities in II-VI semiconductors. Phys. Rev. B 56, 6669-6672 (1997).

54. Ma, Y., Dai, Y., Wei, W., Yu, L. \& Huang, B. Novel Two-Dimensional Tetragonal Monolayer: Metal-TCNQ Networks. J. Phys. Chem. A 117, 5171-5177 (2013).

55. Huang, P. et al. Differential toxicity of $\mathrm{Mn}^{2+}$ and $\mathrm{Mn}^{3+}$ to rat liver tissues: Oxidative damage, membrane fluidity and histopathological changes. Exp. Toxicol. Pathol. 64, 197-203 (2012).

56. Eastmond, D. A., MacGregor, J. T. \& Slesinski, R. S. Trivalent Chromium: Assessing the Genotoxic Risk of an Essential Trace Element and Widely Used Human and Animal Nutritional Supplement. Crit. Rev. Toxicol. 38, 173-190 (2008).

57. Carrero-Sánchez, J. C. et al. Biocompatibility and Toxicological Studies of Carbon Nanotubes Doped with Nitrogen. Nano Lett. 6, 1609-1616 (2006).

58. Wu, M., Zeng, X. C. \& Jena, P. Unusual Magnetic Properties of Functionalized Graphene Nanoribbons. J. Phys. Chem. Lett. 4, 2482-2488 (2013).

59. Kresse, G. \& Furthmüller, J. Efficient iterative schemes for ab initio total-energy calculations using a plane-wave basis set. Phys. Rev. B 54, 11169-11186 (1996).

60. Dudarev, S. L., Botton, G. A., Savrasov, S. Y., Humphreys, C. J. \& Sutton, A. P. Electron-energy-loss spectra and the structural stability of nickel oxide:An LSDA+U study. Phys. Rev. B 57, 1505-1509 (1998).

61. Alling, B., Marten, T. \& Abrikosov, I. A. Effect of magnetic disorder and strong electron correlations on the thermodynamics of CrN. Phys. Rev. B 82, 184430 (2010).

62. Sato, K. et al. First-principles theory of dilute magnetic semiconductors. Rev. Mod. Phys. 82, 1633-1690 (2010).

63. Zhou, J., Wang, Q., Sun, Q., Kawazoe, Y. \& Jena, P. Strain-Induced Spin Crossover in Phthalocyanine-Based Organometallic Sheets. J. Phys. Chem. Lett. 3, 3109-3114 (2012).

64. Zhou, J. \& Sun, Q. Absorption induced modulation of magnetism in twodimensional metal-phthalocyanine porous sheets. J. Chem. Phys. 138, 204706-204707 (2013).

65. Baroni, S., de Gironcoli, S., Dal Corso, A. \& Giannozzi, P. Phonons and related crystal properties from density-functional perturbation theory. Rev. Mod. Phys. 73, 515-562 (2001).

66. Paolo, G. et al. QUANTUM ESPRESSO: a modular and open-source software project for quantum simulations of materials. J. Phys. Condens. Matter 21, 395502 (2009).

\section{Acknowledgments}

This work is partially supported by grants from the National Natural Science Foundation of China (NSFC-11174014, NSFC-21273012), the National Grand Fundamental Research 973 Program of China (Grant No. 2012CB921404), and the Doctoral Program of Higher Education of China (20130001110033).

\section{Author contributions}

Q.W. designed the project; S.Z. performed the calculations; S.Z., Y.L., T.Z. and Q.W analyzed the results and wrote the manuscript.

\section{Additional information}

Supplementary information accompanies this paper at http://www.nature.com/ scientificreports

Competing financial interests: The authors declare no competing financial interests.

How to cite this article: Zhang, S.H., Li, Y.W., Zhao, T.S. \& Wang, Q. Robust ferromagnetism in monolayer chromium nitride. Sci. Rep. 4, 5241; DOI:10.1038/srep05241 (2014). 
(i) This work is licensed under a Creative Commons Attribution-NonCommercialNoDerivs 3.0 Unported License. The images in this article are included in the article's Creative Commons license, unless indicated otherwise in the image credit; if the image is not included under the Creative Commons license, users will need to obtain permission from the license holder in order to reproduce the image. To view a copy of this license, visit http://creativecommons.org/licenses/by-nc-nd/3.0/ 